\title{
INTERVENTION SOCIALE FACE À LA MALADIE: la place du chercheur \\ Maryvonne Charmillot*
}

\begin{abstract}
Résumé: A partir d'une recherche sur la socialisation et le lien social en contexte africain autour du phénomène du sida, cet article propose une réflexion sur la place du chercheur face à la maladie. Comment ce dernier définit-il son rapport à l'objet d'étude, aux informateurs et plus largement aux acteurs concernés par la recherche? Comment, face à la maladie, au sida en particulier, le chercheur gère-t-il les principes méthodologiques telle que la neutralité axiologique? Parvientil à concilier ces derniers avec son engagement moral, social, politique? Ces questions sont traitées à partir des propositions théoriques de Norbert Elias sur l'engagement et la distanciation reprises par Didier Fassin pour analyser les recherches produites en sciences sociales sur le sida en Afrique.
\end{abstract}

Mots-clés: intervention, maladie, place du chercheur, sida, Afrique de l'Ouest.

\section{Cadrage}

Les réflexions développées dans cet article concernent la problématique de l'intervention. Dans son acception ordinaire, intervenir signifie "prendre part à une action, à une affaire en cours dans l'intention d'influer sur son déroulement" (Petit Robert). Parler d'intervention dans les sciences sociales revient communément à chercher comment prendre part à, ou initier des actions dans tel ou

* Université de Genève, Faculté de Psychologie et des Sciences de l'Éducation Artigo recebido em 25 maio 2003; aprovado em 30 ago. 2003. 
tel domaine de la vie sociale avec l'objectif de modifier les choses en cours, de les améliorer. Dans cet article, la perspective adoptée est d'ordre critique; elle est reliée à la place du chercheur, à son rapport à l'objet d'étude, aux informateurs et plus largement aux acteurs concernés par la recherche. Les questions que je désire traiter sont les suivantes: comment, en tant que chercheur, définir son rapport à l'objet d'étude? Comment penser sa relation avec les informateurs? Comment concilier neutralité axiologique et engagement moral, social, politique?

Ces questions sont suggérées par une recherche sur la socialisation et le lien social face au sida réalisée dans une ville moyenne de l'Ouest africain, Ouahigouya, au Burkina Faso. L'objectif est d'examiner la mise à l'épreuve de la solidarité dans un contexte où les transformations économiques, démographiques et sociales relatives à l'épidémie s'inscrivent dans des systèmes sociaux en pleine mutation en raison du processus de modernisation en cours. Loin de lire la situation de crise dans la seule perspective alarmiste de déstructuration des liens familiaux et sociaux, l'intention est de mettre en évidence, au niveau individuel et collectif, les formes d'adaptation développées face aux conséquences de la maladie. Que devient le lien social dans une situation de crise? Comment est-il recomposé? Quelle est la place de la socialisation dans ces recompositions? Telles sont les principales questions qui orientent cette recherche ayant fait l'objet d'une thèse de doctorat en Sciences de l'Éducation à l'Université de Genève (Charmilot, 2002).

Sur le plan théorique, l'adoption du point de vue interactionniste social (en référence aux travaux de Bronckart notamment) a permis de penser le lien social et sa transmission audelà des oppositions entre les courants structuro-fonctionnalistes d'un côté et les courants développés à partir de l'interactionnisme symbolique de l'autre. Le poids de l'Histoire est observé en même temps que la capacité à innover des acteurs sociaux. A partir de cette conception dialectique entre activité collective et action individuelle, la socialisation est envisagée comme un double processus à travers lequel les acteurs rencontrent des conceptions et des pratiques du 
lien social et sont habilités à en produire. Cette orientation a donné lieu à une hypothèse théorique qui a guidé le questionnement susmentionné : face aux processus de dé-liaison causés par le sida, un travail collectif de reconstruction du tissu social se met en place au quotidien. Ce travail collectif, perceptible à travers l'articulation de pratiques individuelles, traduit les capacités d'innovation des acteurs sociaux. Les innovations produites, enfin, ont leur ancrage dans l'histoire.

Sur le plan méthodologique, trois phases d'enquête ont été réalisées. En premier lieu, une série d'entretiens, individuels et collectifs, auprès de personnes âgées, dans le but de connaître les dimensions du lien social propres au contexte, et de mettre en évidence les désordres de l'activité collective intervenus au cours du temps. Les vieux étaient invités à raconter comment ils avaient vécu, comment se passait la vie quotidienne dans le passé, quels étaient les changements perçus aujourd'hui. L'hypothèse secondaire relative à cette première phase d'enquête supposait que si le sida constituait effectivement un facteur de désordre de l'activité collective, il apparaitrait dans les discours sans qu'il ne soit besoin de l'introduire. Cette hypothèse revêt un caractère particulier en regard de la réflexion proposée puisqu'elle traduit la volonté de ne pas imposer l'objet, autrement dit d'intervenir le moins possible dans les récits des informateurs. Suivant les propositions de Laplantine (1995, p. 186), il s'agit "de faire advenir avec les autres ce qu' on ne pense pas, plutôt que de vérifier sur les autres ce qu'on pense".

La seconde phase d'enquête a consisté en une série d'entretiens avec les membres d'une association locale dispensant des soins à domicile à des malades atteints du sida. Créée en 1992 pour lutter contre la malnutrition et contribuer au bien-être de l'enfant, cette association a peu à peu cherché à prendre en compte les problèmes liés à l'apparition du sida. Au travers de séances de sensibilisation à propos de la maladie et ses modes de transmission, les membres notèrent que la première difficulté qui se pose aux malades est l'intégration au sein de la famille et plus largement au sein de la communauté. C'est de ce constat qu'a germé l'initiative des soins à 
domicile. Travailler avec les membres de cette association dans le cadre de ma recherche comprenait un double objectif: a) recueillir leurs observations concernant les relations des patients avec leur entourage et, plus globalement, celles des familles avec la communauté; b) préparer mes visites et les entretiens prévus dans les familles. Ces entretiens dans des familles directement concernées par le sida constituaient en effet, initialement, la troisième phase d'enquête. Ils devaient permettre de mettre en évidence la place de la maladie dans la vie quotidienne et les gestes entrepris par les acteurs concernés face aux ruptures du lien social qu'elle occasionnait. Il est à souligner que je connaissais l'association et plusieurs de ses membres depuis quelques années et que l'accompagnement des agents de santé dans les familles avait été décidé conjointement lors d'une phase exploratoire menée en 1998. Cependant, pour des raisons de conjonctures financières défavorables, ces entretiens n'ont pas pu être réalisés. En effet, les activités de soins à domicile ont été suspendues pendant mon séjour sur place, dans l'attente d'un renouvellement du financement du projet de développement auquel elles étaient liées. Or, comme le note Dozon (1995) il est éthiquement nécessaire de contacter des personnes atteintes de la maladie à travers des institutions qui ont proposé un dépistage aux patients et qui se sont engagées à assurer une prise en charge thérapeutique et psychosociale ultérieure. La suspension contrainte des activités de soins de l'association ne permettait plus cette exigence.

Une troisième phase a dès lors consisté en une série d'entretiens collectifs avec des jeunes. Ce choix a été motivé par les discours des vieux d'une part, dans lesquels les jeunes étaient souvent cités, et le rôle primordial de ces derniers dans la (re)construction du tissu social. En effet, comme le note Becker en introduction de l'ouvrage collectif Vivre et penser le sida en Afrique (1999, p. 13), de nombreuses études, qu'elles se préoccupent du sida ou non, mettent en évidence le fait que les femmes et les jeunes constituent souvent les principaux acteurs des transformations sociales et familiales actuelles. Dans cette perspective, les jeunes étaient invités à parler de leur vie actuelle et de leurs projets d'avenir. Comme dans les entretiens avec les vieux, je m'attendais à voir apparaître le sida comme un facteur de désordre. 
Une hypothèse secondaire supplémentaire avait été formulée, qui exprimait des tensions entre les vieux et les jeunes autour du lien social, tensions exacerbées par la présence du sida et les contraintes nouvelles imposées par la maladie.

\section{Pourquoi penser l'intervention}

Les raisons qui m'ont conduite à considérer avec attention la problématique de l'intervention sont liées au déroulement de la recherche. Au cours des six mois de travail sur le terrain en effet, les interrogations sur ma démarche et mon statut de chercheur ont été multiples. Tiraillée entre les enjeux et les exigences relatives à "l'objet thèse" et certaines réalités locales, j' ai éprouvé des difficultés à me situer. Les questions concernant l'intervention m'ont prise, si j'ose dire, par surprise. Qu'est-ce à dire? Lors de la définition initiale de la recherche, la question de l'intervention n'avait pas été réellement problématisée. Elle était traitée en termes d'enjeux pratiques accompagnant les enjeux théoriques. Autrement dit, la recherche était envisagée comme une recherche de type fondamental dont les résultats pouvaient servir à produire des actions, soit médicales (soins), soit éducatives (prévention, formation), soit encore sociales (assistance des personnes âgées, des orphelins par exemple) adaptées au contexte, c'est-à-dire qui tiennent compte des spécificités économiques et sociales du lieu d'une part, et des façons d' agir et de réagir des acteurs face aux conséquences du sida d'autre part. Telle était la part d'intervention initiale intégrée aux objectifs de la recherche: l'utilité potentielle des résultats envisagés légitimait ma démarche. Concernant l'enquête à proprement parler, les questions avaient été davantage formulées en terme de faisabilité (prises de contact, planification des entretiens, modalités des rencontres: entretiens individuels, de groupes...). Elles avaient trouvé des réponses à travers des recherches antérieures menées dans le contexte choisi, ${ }^{1}$ de même qu'à travers une phase exploratoire lors de laquelle j' avais défini, en accord avec les membres de l'association locale dispensant des soins à domicile, les modalités d'une partie de mon enquête. Je prendrais contact avec des personnes ou des familles concernées par le sida en accompagnant 
les agents de santé lors de leurs visites à domicile. La collaboration envisagée légitimait mes intentions.

La vision quelque peu dichotomique entre enjeux théoriques et enjeux pratiques relative à cette définition initiale de la recherche ne tarda cependant pas à montrer ses limites. J'assumais mal d'être "seulement" un chercheur tentant de produire un document qui pourrait "peut-être" servir. Voici décrites mes incertitudes à travers deux extraits de correspondances adressées à mes collègues:

Je suis chaque jour confrontée à mon statut de chercheur que je ne gère pas (si) facilement. Face à toutes les choses à faire ici, à tous les problèmes de base qui se posent pour tellement d'habitants, face aux malades couchés dans des cours démunies, j'ai de la peine à donner à ma démarche un sens qui soit intégré au contexte. Je me dis: mais tu fais quoi, là, avec ton magnéto de poche et ton micro? Je ressens cela aussi parce que les membres de l'association par l'intermédiaire desquels je réaliserai une partie de mon enquête sont pris dans le quotidien et le stress des dizaines d'activités que gère en parallèle l'association. Il n'y a donc jamais de temps pour discuter, échanger, pour faire se rencontrer nos questionnements respectifs. Aucun élément d'une recherche-action ou de quelque chose comme "recherche et intervention". ${ }^{2}$ (correspondance du 5 mai 2000)

Il est 18 heures. Je traverse la ville, lentement. Je voudrais rouler des kilomètres durant, me concentrer sur la route seulement, arrêter le temps, mettre tout entre parenthèses. Je sais que je peux préparer les visites dans les familles, mais réussirai-je à mener les entretiens prévus? Est-ce que j'aurai la force? Et surtout, surtout, est-ce que j'aurai la conviction de mon - entreprise? Est-ce que c'est bien de faire cela? (correspondance du 23 mai 2000)

Les perspectives d'une thèse "conventionnelle" s'estompent. D'une part parce que, si je ne parviens pas à accumuler d'autres données, ce que j'ai accumulé jusqu'à présent n'est pas suffisant. Et d'autre part, parce que je ne sais pas sous quelle forme les restituer. (...) Je ne me sens pas à l'aise, pour le faire, avec le langage scientifique. Parce que je ne voudrais pas me voiler la face, c'est-à-dire, en quelque sorte, utiliser le langage savant et rester faussement neutre, ne pas prendre position contre un système qui semble trouver ce continent plus encombrant qu'autre chose. L'accès quasi impossible aux médicaments donne à réfléchir à un vieux qui me disait que le sida était une maladie 
des Blancs pour tuer les Africains. Ce que je dis manque probablement de nuances, mais il me semble qu'il y ait là des éléments essentiels à travailler en tant que chercheur. (correspondance du 21 juin 2000)

Parallèlement aux questions soulevées dans ces extraits questions qui prennent place dans le projet global de recherche, dans ses objectifs et dans la démarche qui lui est attachée -, s'ouvre un espace plus circonscrit, celui du recueil des données. En l'occurrence, dans la recherche présentée ici, l'espace des entretiens. Dans cet espace également, se sont posées une série de questions relatives à l'intervention, plus précisément au rapport entre chercheur et informateurs au moment des échanges. La technique de l'entretien avait été retenue pour son inscription dans la démarche compréhensive (Schurmans, 2003), autrement dit en tant qu'outil permettant de construire l'objet à partir du point de vue des informateurs. Je me positionnais de ce fait au-delà de certains principes méthodologiques (ou en opposition) assignés à l'entretien, tels que distance et non engagement du chercheur, sachant que les données produites au cours de l'échange résultent d'une co-consctruction entre chercheur et informateurs (Blanchet, 1985). Mes représentations du dispositif d'enquête, inspirées des propositions de Kaufmann (1996), étaient donc davantage portées vers l'ouverture et la souplesse des interactions, plutôt que vers une rigidité des rôles et des prises de paroles des acteurs en présence. Lors de l'enquête néanmoins, deux scénarios m'ont particulièrement interpellée. Le premier renvoie à un renversement des rôles entre enquêteur et informateurs : les jeunes interviewés ont par moment posé de nombreuses questions à l'interviewer, donnant à ce dernier le sentiment de ne plus tenir son rôle. Comment, dès lors, répondre aux informateurs? Le second concerne l'attitude à adopter, en tant qu'interviewer, face à des propos concernant le sida exprimés par les personnes interviewées et jugées erronés, inexacts ou partiels par le chercheur. Doit-il intervenir, et si oui, comment le faire tout en respectant les spécificités des échanges propres à l'entretien de recherche? Dans mes notes de terrain, j'exprime ainsi ce double questionnement:

Un aspect prononcé dans les entretiens lorsque j' aborde le sida, c'est le renversement des positions: c'est à l'interviewer qu'on pose des 
questions; le chercheur devient lui-même, en quelque sorte, l'interviewé. Si cela se passe en fin d'entretien, ça ne pose pas trop de problèmes, mais si cela se produit plus tôt, il faut savoir rétablir la situation, étant donné que l'objectif de l'entretien est avant tout de recueillir de l'information, et pas tant d'en donner.

Une autre question à laquelle me confrontent certains entretiens, c'est la position de l'interviewer face aux dires des interviewés. Lorsque des jeunes sont peu au clair sur les modes de transmission et affirment par exemple qu' "il vaut mieux fuir la personne [atteinte du sida] parce qu'on ne sait jamais", que doit faire l'interviewer? Intervenir, soit pendant l'entretien, soit à la fin, en disant quelque chose du genre: "Au fait, concernant les modes de transmission, il faut savoir que..."? Ou s'en tenir aux propos recueillis? (Journal de terrain, p. 95-96)

Réfléchissant à ces questions durant l'enquête et à l'issue du travail de terrain, je me suis aperçu qu'elles avaient été générées par un point spécifique de l'objet d'étude, à savoir le sida. En effet, de telles questions ne se sont jamais posées lorsque les informateurs évoquaient la vie quotidienne ou me décrivaient, par exemple, les liens familiaux. Concernant la première de ces questions, relative aux renversements de position entre l'interviewer et l'interviewé, je me suis efforcée de répondre au mieux aux questions qu'on m'adressait (est-ce que le sida existe en Suisse/France? est-ce qu'en Suisse/France, il y a un vaccin? quels sont les médicaments à disposition? est-ce vrai que le sida se trouve dans les capotes? est-ce que vous allez nous donner des informations sur le sida?). J'aurais néanmoins souhaité des outils me permettant de gérer cette dynamique et mon rapport aux personnes interviewées sans crainte de voir le recueil de données mis en péril. Car, dans ma vision des choses, les questions des interviewés témoignaient d'un relatif échec de l'entretien d'une part (l'interviewer ne sachant pas tenir son rôle), et n'apportaient pas les informations recherchées d'autre part (dans le sens des hypothèses). Au moment de l'analyse des données, j'ai considéré les choses différemment, en prenant en compte les questions des informateurs comme des données, c'est-à-dire comme des éléments qui informent - au même titre que les réponses - sur la question de recherche et les hypothèses. Autrement dit, en évitant de "reléguer" les questions des interviewés en fin d'entretien mais en interrogeant leur sens en regard de la problématique. 
La seconde question, relative à la position de l'interviewer face à certaines affirmations des interviewés, met également en cause le rôle de l'enquêteur qui, habituellement, ne commente ni ne juge ce que les informateurs lui racontent, lui dévoilent ou lui expliquent. Cette question, relative ici à l'objet sida, aurait pu apparaître de façon identique lors d'une recherche précédente dans le même lieu. Je m'étais, dans le cadre d'une autre recherche, entretenue avec des femmes à propos des maladies de leurs enfants. Ces femmes étaient invitées à s'exprimer librement sur les problèmes de santé auxquels elles étaient confrontées. Les trois thèmes guidant l'échange étaient la symptomatologie (à quoi remarque-t-on la maladie?), l'étiologie (quelles sont les causes de la maladie?) et la thérapeutique (comment soigner la maladie?) La catégorie nosographique la plus évoquée par les femmes était la "maladie de l'oiseau", maladie présentant de fortes similitudes avec le paludisme, et dont la cause est imputée à un oiseau maléfique. Connaissant le danger représenté par les moustiques à propos de cette maladie, j' aurais pu, par exemple, demander aux femmes si leurs enfants dormaient sous une moustiquaire et introduire, par ce biais, quelques informations sur les causes de certaines des fièvres qu'elles me décrivaient. La question ne s'est pourtant jamais posée. Pourquoi? Pourquoi sentir une responsabilité, en tant qu'interviewer, face à des personnes exposées aux risques du sida? Pourquoi ne pas sentir cette responsabilité face aux risques de la malaria? L'hypothèse que je formulerais est relative aux images et aux discours véhiculés à propos des différentes maladies présentes sur le lieu de l'enquête. Le sida, par exemple, est toujours évoqué dans une perspective alarmiste ("le drame du sida"). Chacun se doit d'être concerné, non seulement pour soi-même mais aussi pour les autres, par ce "terrible fléau qui ravage le continent africain". Le paludisme, quant à lui, est plus rarement décrit comme une menace (lorsqu'il n'est pas passé sous silence), quand bien même il a entraîné pendant des années plus de décès que le sida. Je ne débattrai pas ici de ces différences de traitement des phénomènes. J'ajouterai simplement, pour compléter mon hypothèse, que l'image "officielle" (Latouche, 1998) construite à propos du sida en Afrique, par les médias autant que par certaines recherches concourt peut-être aux 
développements de réflexions concernant l'intervention - en tant qu'actions entreprises pour modifier le cours des choses - que d'autres maladies légitimeraient tout autant.

\section{Comment penser l'intervention}

Si j'ai choisi de me pencher sur la problématique de l'intervention, c'est, comme je l'ai écrit plus haut, en raison de certaines difficultés rencontrées sur le terrain. Et si j'ai choisi de prendre au sérieux ces difficultés, c'est en raison de l'orientation théorique et de la perspective épistémologique adoptées. En effet, le rapport à l'objet, aux informateurs et plus largement aux acteurs concernés par la recherche n'est pas pris en compte de la même manière par toutes les tendances théoriques. Du côté du structurofonctionnalisme, qui a été écarté de mes options pour la place prépondérante donnée aux déterminismes et la trop faible prise en compte des actions des individus, la position de l'observateur est extérieure au social. Autrement dit, ce dernier n'est impliqué ni de près, ni de loin, dans les phénomènes portés à la connaissance scientifique. Parmi les courants opposés développés à partir de l'interactionnisme symbolique, écartés, à l'inverse, en raison du peu d'intérêt porté au structurel et à l'Histoire, l'observateur agit à l'intérieur de la situation étudiée et participe à la production des résultats au même titre que les autres acteurs. Dans le champ théorique dialectique adopté, celui de l'interactionnisme social, la place du chercheur n'est ni totalement extérieure, ni totalement participante. Ce dernier se doit de la définir, de même qu'il doit déterminer son rapport avec les informateurs. "Il incombe au chercheur", écrit MarieNoëlle Schurmans (2001), "d'élucider la place qu'il adopte entre extériorité surplombante et participation directe à l'effervescence sociale. Il s'agit là, pour lui, de définir son rapport avec ses informateurs. Ce qui implique, au-delà de la phase de récoltes de données, de définir sa part de responsabilité quant aux résultats de ses recherches, la façon dont ils seront restitués à ceux qui en sont à la source, la validité que ceux-ci accorderont à ses propos”. 
Pour tenter de définir la place du chercheur, ou pour le moins tenter de problématiser les questionnements évoqués à l'issue du travail de terrain, je m'appuierai sur un article de Didier Fassin (1999, p. 46) dans lequel ce dernier, médecin et anthropologue, analyse la position des chercheurs face à leur objet d'étude, le sida en particulier. La question du rapport à l'objet demeure, à ses yeux, 'insuffisamment réfléchie, alors même qu'elle concerne le travail quotidien des chercheurs et la légitimité de leur intervention dans le monde social". Cette question du rapport à l'objet se pose selon lui de deux manières étroitement liées entre elles mais aux enjeux distincts: quelle connaissance objective peut-on produire sur un monde d'objets dont on fait soi-même partie (enjeu épistémologique); quelle position axiologique peut-on défendre dans l'étude de phénomènes mettant en jeu des valeurs sur lesquelles on porte soi-même des jugements (enjeu politique)? Pour tenter de répondre à ces questions, Fassin s'appuie sur la théorie de Norbert Elias concernant l'engagement et la distanciation, deux opérations intellectuelles à la fois indissociables et contradictoires qui déterminent le cours des actions humaines. Dans le sens commun, l'engagement et la distance sont conçues séparément. Un chercheur engagé est un chercheur présent sur la scène sociale et la définition de l'engagement est de nature politique. La distance quant à elle se rapporte à l'objet et sa nature est davantage épistémologique. Chez Elias, engagement et distanciation se situent simultanément sur les plans politiques et épistémologiques et présentent de la sorte l'avantage de rendre mieux compte de la réalité que ne le faisaient notamment les approches de Durkheim et de Weber. La position du premier est en effet ambiguë puisque d'un côté, le sociologue prône la rupture avec les prénotions et, de l'autre, il produit des analyses sur la société de son époque non exemptes de jugements de valeur. "La thèse éléasienne de l'indissociabilité des dimensions épistémologique et politique de la relation des sciences sociales à leur objet permet de dépasser l'illusion positiviste que contredit, dans les faits, le discours normatif. Le chercheur est engagé par rapport au monde qu'il étudie à la fois comme sujet connaissant et comme sujet citoyen" (p. 43). La théorie d'Elias permet également de dépasser l'opposition de Weber entre le savant et le politique. Pour le sociologue 
allemand en effet, si le savant peut avoir des convictions personnelles, il ne doit en aucun cas les mêler à ses recherches ou les enseigner. L'action du politique, à l'inverse, est déterminée par des jugements de valeur, même s'il a recours, pour prendre ses décisions, aux connaissances disponibles dans son domaine d'intervention. Là encore, la théorie d'Elias permet de penser la tension entre l'engagement et la distanciation, plutôt que de les considérer comme deux entités séparées.

Fassin fait néanmoins ressortir un paramètre que la théorie d'Elias ne met pas en évidence: la diversité des formes d'engagement et de distanciation. Cette diversité correspond à la multiplicité des paradigmes présents dans les sciences sociales. Pour Fassin, la prise en considération de cette diversité est fondamentale car elle permet de quitter le niveau de généralité de la théorie d'Elias pour mettre en évidence la singularité des pratiques et les situer historiquement. Pour en rendre compte, il identifie deux dimensions: le registre de l'action et le registre de l'analyse. Le registre de l'action va de la recherche appliquée (engagement) à la recherche fondamentale (distanciation) et renvoie aux enjeux pratiques définis ou non par le chercheur. Le registre de l'analyse va de l'adhésion (engagement) à la critique (distanciation) et concerne les enjeux de connaissance: le chercheur reproduit-il les modèles théoriques communément sollicités ou tentet-il de développer de nouveaux cadres interprétatifs? Ce croisement des registres et des positions peut être représenté schématiquement dans un tableau à double entrée. Pour ne pas risquer, cependant, de figer les positions, je mets en évidence par des flèches le rapport de tension qui les lie.

\section{Tableau 1 - Formes d'engagement et de distanciation}

\begin{tabular}{|c|c|}
\hline position & ENGAGEMENT \\
\hline ACTION & recherche appliquée $\longrightarrow$ DISTANCIATION \\
\hline ANALYSE & adhésion $\longrightarrow$ recherche fondamentale \\
\hline
\end{tabular}




\section{Comment penser l'intervention dans les recherches traitant du sida?}

Quelle sont les implications de l'objet maladie concernant l'intervention? Qu'en est-il du sida? Pour tenter de répondre à ces deux questions, c'est toujours à Didier Fassin que je me référerai. Tenant compte du modèle d'Elias, en y ajoutant les dimensions de l'action et de l'analyse, il dresse une topographie des recherches sur le sida effectuées en Afrique, en distinguant deux périodes.

Au cours de la première période, qui se situe vers le milieu des années 80 et dure quelques années, on parle d' "urgence anthropologique" et le niveau d'exigence théorique et méthodologique est faible: "Les frontières entre la médecine et l'anthropologie s'effacent, les médecins se hasardent volontiers à des analyses approximatives de faits sociaux et culturels, alors que les anthropologues s'efforcent de raisonner avec des schémas empruntés à l'épidémiologie et à la biologie. Le statut des énoncés scientifiques devient alors très incertain" (p. 47). Le thème de la "promiscuité sexuelle" et l'hypothèse d'une "sexualité africaine déviante" comme facteur explicatif exclusif de l'épidémie sont révélateurs du statut de ces premières recherches en sciences sociales sur le sida en Afrique. Il n'y a plus de distance, ni épistémologique, ni politique: "La particularité de ces pionniers est (...) d'avoir réduit de manière extrême la distance à l'objet en ne mettant pas en œuvre les procédures habituelles de construction scientifique et en acceptant sans les critiquer les idées que leur proposait le sens commun. Pour utiliser un langage éléasien, tout se passe comme si les barrières de l'autocontrôle au sein de tout champ scientifique en temps de fonctionnement normal s'étaient brutalement abaissées: plus de prévention à l'égard des a priori, en particulier culturalistes; valeurs et jugements s'expriment sans leur garde-fous habituels; on assiste à une sorte de décivilisation de la recherche" (p. 50-51).

La seconde période commence à partir de 1988 où l'on assiste à une diversification des recherches. Toujours en fonction du modèle rapportant l'engagement et la distanciation sur l'axe de l'action d'une 
part et sur l'axe de l'analyse d'autre part, Fassin identifie trois positions. La première est la position appliquée. Les recherches les plus classiques qui s'y rattachent sont les enquêtes communément nommées CACP (connaissances, attitudes, croyances et pratiques) et les enquêtes sur le comportement sexuel. La connaissance de ces différents aspects doit servir l'élaboration de programmes de prévention et par conséquent améliorer la santé publique. La légitimité de ces recherches repose d'une part, dans le champ scientifique, sur "la solidité supposée des données quantitatives uniformes obtenues dans de nombreux pays par des procédures éprouvées d'échantillonnage, de recueil et d'analyse" et d'autre part, dans le champ de la santé publique, sur "la conviction de l'adéquation existant entre le problème posé et le dispositif d'enquête mis en place" ( $\mathrm{p}$. 53). Les critiques qui ont été adressées à ces recherches concernent la fiabilité des formulations inhérentes au questionnaire, ainsi que la centration sur les aspects sexuels au détriment d'autres facteurs. Fassin, quant à lui, montre que la lecture anthropologique de l'épidémie proposée par la position appliquée est moins distante qu'il n'y paraît: “(...) derrière l'apparente distance introduite par la quantification des données recueillies et l'énumération des références ethnographiques, la sélection à laquelle procède l' anthropologue pour étayer sa démonstration témoigne-t-elle d'une toujours impossible distanciation" (p. 54).

La seconde position est une position critique. Elle se divise en trois orientations: a) les recherches qui discutent les présupposés sur lesquels sont basées les études relatives aux groupes et comportements à risque; ces présupposés ont des effets négatifs sur les programmes de prévention; b) les recherches qui reposent sur l'analyse des structures sociales et politiques; certaines pratiques, comme la prostitution par exemple, sont moins culturelles que liées à des contraintes économiques et sociales de plus en plus fortes s'exerçant sur les femmes; c) les recherches, enfin, qui montrent les effets des politiques économiques et sanitaires mondiales sur le sida. Suivant le modèle du rapport aux axes de l'action et de l'analyse, les chercheurs de la position critique devraient présenter une double mise à distance: par "le regard critique sur les faits et leur interprétation" d'une part, 
par "le refus de s'associer à des pratiques de recherche et de prévention dont ils refusent les présupposés" d'autre part. Qu'en est-il en réalité? Pour répondre, Fassin s'appuie sur la critique de deux auteurs adressée aux médecins et aux chercheurs (Packard \& Epstein, 1991). Par rapport à l'analyse, la mise en évidence de causes sociales plutôt que culturelles ne garantit pas forcément l'objectivité (la distanciation). Par rapport à l'action, refuser de s'inscrire dans des programmes de prévention qui "encouragent la culpabilisation" des victimes de la maladie et prôner un travail sur les facteurs qui accélèrent l'évolution de cette dernière - la malnutrition et la paupérisation, par exemple -, n'est pas sans rapport avec l'action. Les chercheurs de la position critique ne sont donc ni totalement objectifs, ni tellement éloignés de l'action. Leur engagement, écrit Fassin (1999, p. 58), "prend seulement un autre forme, moins immédiate et plus détachée".

La troisième position est l'implication. De quoi s'agit-il? 'Le sida a entraîné un changement chez bien des anthropologues. Autour de cette maladie, s'est défini un autre rapport à l'engagement, une nouvelle implication des chercheurs. Tant dans l'analyse des multiples figures du rapport des individus et des groupes sociaux à l'Autre que dans la pratique du terrain qui fonde leur métier, les anthropologues ont dû passer d'une approche qui pouvait se permettre la distanciation, à une confrontation très directe, à une responsabilité envers les malades. Pour l'anthropologue aussi, le sida a valeur de 'crise'. Et cette crise touche la relation qu'il noue avec son terrain, avec les personnes avec lesquelles il travaille. Le repositionnement du chercheur, souvent considéré comme 'ayant un savoir', est difficile à définir dans tous les contextes, mais la distanciation neutre n'est avec le sida ni possible, ni humainement acceptable: c'est l'implication qui est nécessaire” (Benoist \& Desclaux, 1996, cités par Fassin, 1999, p. 59).

Fassin, tout en notant que l'implication a engendré une nouvelle modalité de l'engagement et de la distanciation, se demande pourquoi d'autres maladies, comme le paludisme ou le cancer n'ont pas eu cet impact sur les pratiques des chercheurs comme sur celles des médecins. La question de l'engagement et de la distanciation se pose, selon lui, 
dans l'implication, de la manière suivante: "Comment associer la préoccupation de défense des malades - autrement dit, l'engagement dans l' arène sociale - et le souci de conserver une posture scientifique - c'est-à-dire le maintien d'une distanciation par rapport aux jugements de valeur?" (p. 60). Deux chercheurs, Marc-Eric Gruénais et Laurent Vidal, ont, par exemple, constaté que le moment de l'annonce de la séropositivité était souvent éludé par les médecins, alors que c'est un moment crucial dans la vie du patient. Comment le chercheur doit-il réagir face à de tels constats? Les différentes réponses apportées par Gruénais et Vidal montrent qu'une attitude plus ou moins engagée ou plus ou moins distanciée contribue chacune à une modification des pratiques. Et Fassin note par ailleurs: “(...) il est intéressant de constater empiriquement que l'engagement dans l'analyse est souvent associé à la distanciation dans l'action, cependant que la distanciation dans l'analyse va fréquemment de pair avec l'engagement dans l'action" (p. 61). Autrement dit, plus le questionnement, la remise en question, sont intenses, plus la part d'action est faible, et vice et versa.

\section{Retour sur la recherche}

Quelle compréhension l'analyse de Fassin permet-elle de produire à propos des questions sous-jacentes aux extraits de correspondance et de notes de terrain soumis à l'analyse? La première question concerne le rapport à l'action: quelle démarche adopter qui serve l'action dans l'immédiat et non à contre-temps, c'est-à-dire après restitution des données? Cette question traduit le malaise ressenti par le chercheur en raison d'un décalage qu'il perçoit entre sa fonction et celle des acteurs présents autour de lui. Il a le sentiment de réaliser un "exercice intellectuel" dans un contexte d'extrême pauvreté, et cela ôte à ses yeux tout sens à sa démarche. Suivant le modèle de Elias repris par Fassin, le décalage peut être exprimé en termes de rapports à l'action et à l'analyse. Les rapports développés par le chercheur sont très différents des rapports développés par les acteurs qu'il est amené à côtoyer, en l'occurrence ici, les agents de santé. Le chercheur, par la définition initiale de sa problématique, occupe une position de distanciation tant sur le plan de l'analyse que celui de 
l'action. En effet, d'une part, sa visée première est une visée de connaissance: la problématique du lien social, renouvelée à l'aune de l'interactionnisme social, marque une distanciation face aux modèles théoriques classiques. Pour reprendre les termes de Fassin (voir Tableau 1), il n'y a pas adhésion aux théories communément sollicitées pour traiter la problématique du lien social, mais critique. D'autre part, les enjeux pratiques définis dans la recherche sont secondaires: des activités pourront ou non être mises en place suite aux constats mis en évidence, et sans la participation du chercheur. La distanciation est donc également de mise sur le plan de l'action. Autrement dit, on est plus proche d'une recherche fondamentale que d'une recherche-action. Les acteurs du projet de soins à domicile que côtoie quotidiennement le chercheur sont au contraire fortement engagés sur les deux axes. La visée première de leur projet est une visée pratique d'une part. La prévention et les soins envisagés reposent sur les modèles éprouvés de l'éducation à la santé et de la biomédecine d'autres part.

Pour atténuer son malaise, le chercheur souhaite collaborer avec les agents de santé. Cette collaboration peut être envisagée comme un moyen de générer un équilibre entre le rapport à l'action du chercheur (rapport distant) et celui des autres acteurs (rapport engagé). Elle peut en même temps permettre au chercheur de partager son rapport à l'analyse, autrement dit de faire part de certains de ces questionnements théoriques et épistémologiques. Reste à savoir cependant comment définir et mettre en place cette collaboration pour qu'elle serve en même temps les intérêts de connaissance du chercheur et les préoccupations pratiques des acteurs sur le terrain. Si l'analyse de Fassin permet - préalable indispensable - de circonscrire les positions des acteurs en présence, il reste à modéliser des procédures offrant en parallèle de la connaissance et de l'intervention (au sens de prendre part aux actions en cours dans l'intention d'en transformer le déroulement). Certaines perspectives développées dans le domaine de l'analyse du travail, celle d'Yves Clot en particulier, qui distingue objet d'étude (lié à l'action) et objet de recherche (lié à la connaissance), peuvent proposer des outils pertinents et lever le malaise de l' "exercice intellectuel" décrit plus 
haut. Cette perspective peut également offrir une part de réponse à la seconde question ressortant des extraits de correspondance, qui porte sur la valeur de l'entreprise de recherche (est-ce bien de faire cela), car l'objet d'étude émane toujours d'une demande des acteurs sur le terrain.

Dans le cadre de cette recherche, les réponses théoriques aux questions de l'intervention développées au moment de la rédaction de la thèse ont été accompagnées d'un retour de l'information sur le terrain. Un séjour d'un mois en août 2002, autrement dit deux ans après le travail d'enquête, a permis, d'une part, de poursuivre les échanges engagés avec les membres de l'association, de voir comment les structures de soins avaient évolué, de présenter mon propre travail. Compte-tenu du décalage décrit plus haut entre les finalités de connaissance de ce dernier et l'engagement dans l'action des membres de l'association, ce partage a contribué à une meilleure compréhension mutuelle: achevé, mon travail de recherche devenait en un certain sens plus concret, et je mesurai davantage, au vu des changements survenus (intensification des activités de soins, création d'un centre de dépistage) les conjonctures financières auxquelles peuvent être soumises des associations travaillant dans le domaine de la santé. Ce "retour sur le terrain" a, d'autre part, été complété par la rencontre avec quelques groupes de jeunes ayant participé aux entretiens. Certains d'entre eux avaient reçu, à l'issue du travail de terrain, soit la retranscription, soit une copie audio de ces derniers, et ils avaient poursuivi la "causerie" entre eux. Dans ce cas également, la rencontre a offert une occasion aux informateurs de montrer ce qu' avait suscité en eux les entretiens effectués, et au chercheur de considérer l'impact - la plupart du temps invisible parce que sans intérêt dans la recherche, ou parce qu'un retour aux sources d'informations n'est pas envisageable - que peuvent produire les échanges provoqués au moment de l'enquête.

\section{Conclusion}

La réflexion développée dans cet article à propos de l'intervention met l'accent sur la place du chercheur. Comment ce 
dernier définit-il son rapport à l'objet d'étude et sa relation avec les informateurs? Comment gère-t-il en même temps le principe de neutralité propre à l'activité scientifique et ses engagements sur la scène sociale ou politique? L'analyse construite à partir d'une recherche sur la socialisation et le lien social face au sida met en évidence les difficultés susceptibles d'être rencontrées par le chercheur sur le terrain lorsque son rapport à l'action et à l'analyse n'est pas clairement défini, autrement dit lorsque la place des enjeux pratiques et théoriques n'est pas suffisamment questionnée, en regard à l'objet d'une part, et aux réalités du terrain d'autre part. Car si la réflexion relative à l'intervention vaut pour tout objet de recherche et dans tout contexte, les recherches liées à la maladie dans des contextes où l'urgence prime semblent davantage risquer de ne pas répondre aux critères épistémologiques et déontologiques attachés normalement à l'activité scientifique. La prise au sérieux de la problématique de l'intervention exigée par ce type de recherches peut alors offrir des opportunités de penser l'intervention concernant d'autres objets.

\section{Notas}

1 Un mémoire de licence en sciences de l'éducation à l'Université de Genève (Charmillot, 1997) ainsi qu'une enquête sur la problématique de l'aide mandatée par une ONG franco-suisse (GRAD, 2000).

2 L'expression "recherche et intervention" est utilisée ici en référence à l'une des mentions proposées dans la licence en sciences de l'éducation à l'Université de Genève: Licence Mention Recherche et Intervention (LMRI). Cette formulation, issue du nouveau plan d'étude, témoigne d'une volonté d'intégrer la problématique de l'intervention dans la formation à la recherche.

Resumo: A partir de uma pesquisa sobre socialização e laços sociais a respeito do fenômeno da AIDS, este artigo investiga o papel do pesquisador em relação à doença. Como ele ou ela define sua relação com o objeto de estudo, com os informantes e de forma mais ampla com os atores envolvidos na pesquisa? Como o(a) pesquisador(a) lida com princípios metodológicos como a neutralidade axiológica face à 
doença, particularmente no caso da AIDS? Como ele ou ela busca conciliar este princípio com seu engajamento moral, social e político. Estas questões são discutidas com base nas proposições teóricas de Norbert Elias sobre engajamento e distanciamento, tal como utilizado por Didier Fassin para analisar a pesquisa das Ciências Sociais sobre AIDS na África.

Palavras-chave: intervenção, doença, lugar do pesquisador, AIDS, África Ocidental.

Abstract: Taking as a starting point research in the African context on socialisation and on social links concerning the AIDS phenomenon, this article investigates the role of the researcher in relation to the disease. How does he or she define his or her own relation to the object of study, to the informants and, more widely, to the actors concerned by the research? How does the researcher deal with methodological theories, such as that of moral neutrality towards the disease - in particular that of AIDS? Can he or she manage to accommodate the concept of neutrality with his or her own moral, social and political engagement? These questions are discussed on the basis of theoretical propositions of Norbert Elias concerning commitment and the taking of distance as used by Didier Fassin to analyse social science research on AIDS in Africa.

Key-words: intervention, disease, role of the researcher, AIDS, Western Africa.

\section{Bibliographie}

BLANCHET, A. et al. L'entretien dans les sciences sociales. Paris: Dunod, 1985.

BRONCKART, J. P. Activité langagière, texte et discours: pour un interactionnisme socio-discursif. Lausanne: Delachaux et Niestlé, 1996a.

. S'entendre pour agir et agir pour s'entendre. In: BAUDOIN, J. M.; FRIEDRICH, J. Théories de l'action et éducation. Bruxelles: De Boeck Université, 2001.

BECKER, C.; DOZON, J. P.; OBBO, C.; TOURE, M. Vivre et penser le sida en Afrique. Paris: Karthala, 1999. 
CHARMILLOT, M. Les savoirs de la maladie: éducation à la santé en contexte africain. Cahiers de la Section des Sciences de l'Education, Université de Genève, n. 81, 1997.

. Socialisation et lien social : une étude de cas autour du sida dans la ville de Ouahigouya (Burkina Faso). Genève, 2002. Thèse n. 308, FAPSE.

CLOT, Y. Le travail sans l'homme? : pour une psychologie des milieux de travail et de vie. Paris: La Découverte, 1995.

DOZON, J. P.; VIDAL, L. Les sciences sociales face au sida: cas africains autour de l'exemple ivoirien. Paris: ORSTOM, 1995.

FASSIN, D. L'anthropologie entre engagement et distanciation: essai de sociologie des recherches en sciences sociales sur le sida en Afrique. In: BECKER, C.; DOZON, J. P; OBBO, CH.; TOURE, M. Vivre et penser le sida en Afrique. Paris: Karthala, 1999.

KAUFMANN, J. C. L'entretien compréhensif. Paris: Nathan, 1996.

LAPLANTINE, F. L'anthropologie. Paris: Payot, 1995.

LATOUCHE, S. L'autre Afrique: entre don et marché. Paris: Albin Michel, 1998.

SCHURMANS, M. N. La construction sociale de la connaissance comme action. In: BAUDOIN, J. M.; FRIEDRICH, J. (Eds.). Théories de l'action et éducation. Bruxelles: De Boeck Université, 2001. Les solitudes. Paris: PUF, 2003. 
